\title{
Dietary fish oil and curcumin combine to modulate colonic cytokinetics and gene expression in dextran sodium sulphate-treated mice
}

\author{
Qian Jia ${ }^{1,2}$, Ivan Ivanov ${ }^{3,4}$, Zlatomir Z. Zlatev ${ }^{5}$, Robert C. Alaniz ${ }^{6}$, Brad R. Weeks ${ }^{7}$, Evelyn S. Callaway ${ }^{1}$, \\ Jennifer S. Goldsby ${ }^{1}$, Laurie A. Davidson ${ }^{1,4}$, Yang-Yi Fan ${ }^{1,2}$, Lan Zhou ${ }^{8}$, Joanne R. Lupton ${ }^{1,4}$, \\ David N. McMurray ${ }^{1,4,6}$ and Robert S. Chapkin ${ }^{1,2,4 *}$ \\ ${ }^{1}$ Program in Integrative Nutrition and Complex Diseases, Texas AEM University, College Station, TX, USA \\ ${ }^{2}$ Vegetable Fruit Improvement Center, Texas AGM University, College Station, TX, USA \\ ${ }^{3}$ Department of Veterinary Physiology and Pharmacology, Texas AGM University, College Station, TX, USA \\ ${ }^{4}$ Center for Environmental and Rural Health, Texas AGM University, College Station, TX 77843-2253, USA \\ ${ }^{5}$ Department of Mathematics and Informatics, Sofia University "Kl. Ohrdiski", Sofia, Bulgaria \\ ${ }^{6}$ Department of Microbial and Molecular Pathogenesis, Texas AछM University Health Science Center, College Station, \\ TX 77840, USA \\ ${ }^{7}$ Department of Veterinary Pathobiology, Texas A\&M University, College Station, TX, USA \\ ${ }^{8}$ Department of Statistics, Texas AGM University, College Station, TX, USA
}

(Received 25 October 2010 - Revised 10 January 2011 - Accepted 11 January 2011 - First published online 15 March 2011)

\section{Abstract}

Both fish oil (FO) and curcumin have potential as anti-tumour and anti-inflammatory agents. To further explore their combined effects on dextran sodium sulphate (DSS)-induced colitis, C57BL/ 6 mice were randomised to four diets $(2 \times 2$ design $)$ differing in fatty acid content with or without curcumin supplementation (FO, FO $+2 \%$ curcumin, maize oil (control, $\mathrm{MO}$ ) or MO $+2 \%$ curcumin). Mice were exposed to one or two cycles of DSS in the drinking-water to induce either acute or chronic intestinal inflammation, respectively. FO-fed mice exposed to the single-cycle DSS treatment exhibited the highest mortality ( $40 \%$, seventeen of forty-three) compared with MO with the lowest mortality (3\%, one of twenty-nine) $(P=0.0008)$. Addition of curcumin to MO increased $(P=0 \cdot 003)$ mortality to $37 \%$ compared with the control. Consistent with animal survival data, following the one- or two-cycle DSS treatment, both dietary FO and curcumin promoted mucosal injury/ulceration compared with MO. In contrast, compared with other diets, combined FO and curcumin feeding enhanced the resolution of chronic inflammation and suppressed $(P<0.05)$ a key inflammatory mediator, NF- $\mathrm{kB}$, in the colon mucosa. Mucosal microarray analysis revealed that dietary FO, curcumin and FO plus curcumin combination differentially modulated the expression of genes induced by DSS treatment. These results suggest that dietary lipids and curcumin interact to regulate mucosal homeostasis and the resolution of chronic inflammation in the colon.

Key words: Colitis: Resolution of inflammation: Mucosal repair

Inflammatory bowel diseases (IBD), including ulcerative colitis and Crohn's disease, are chronic relapsing inflammatory conditions of unknown aetiology in the intestinal tract ${ }^{(1,2)}$. Based on the present understanding of the pathogenesis of IBD, activated immune cells can destroy the intestinal barrier either directly via cytotoxicity or indirectly through the release of cytokines, reactive oxygen species and other metabolites ${ }^{(2)}$. In addition, the risk of developing colorectal cancer increases approximately $0 \cdot 5-1 \%$ each year after 7 years in patients with $\mathrm{IBD}^{(3)}$. Despite the well-known functional link between inflammation and colon cancer, the pathways regulating the initiation of colon cancer in the presence of chronic inflammation remain unclear. Therefore, it is important to identify the regulatory mechanisms underlying inflammation and tumorigenesis in the colon.

Dietary long-chain $n$-3 PUFA found in fish oil (FO), specifically EPA $\left(20: 5^{\Delta 5,8,11,14,17}\right)$ and DHA $\left(22: 6^{\Delta 4,7,10,13,16,19}\right)$, are capable of ameliorating critical determinants which link inflammation and cancer development and progression ${ }^{(4-6)}$. In contrast, dietary maize oil (MO) rich in $n-6$ PUFA (found

Abbreviations: COX, cyclo-oxygenase; DSS, dextran sodium sulphate; FO, fish oil; FO-cur, fish oil plus curcumin; IBD, inflammatory bowel diseases; iNOS, inducible nitric oxide synthase; MO, maize oil; MO-cur, maize oil plus curcumin; TUNEL, terminal deoxynucleotidyl transferase-mediated uridine 5 'triphosphate-biotin nick-end labelling.

*Corresponding author: Dr R. S. Chapkin, fax +1979 862 2378, email r-chapkin@tamu.edu 
in vegetable oils), e.g. linoleic acid $\left(18: 2^{\Delta 9,12}\right)$ and arachidonic acid $\left(20: 4^{\Delta 5,8,11,14}\right)$, is considered as a pro-inflammatory dietary component that enhances the development of colon tumours $^{(7,8)}$. To date, the effects of $n$-3 PUFA on susceptibility to colitis and colon cancer have not been determined, and a unifying mechanistic hypothesis addressing how n-3 PUFA selectively modulate colonic inflammation is lacking. Furthermore, it has been demonstrated that $n-3$ PUFA can inhibit

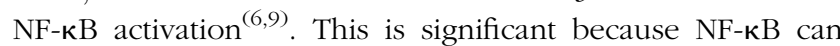
modulate several steps in the inflammatory cascade by inducing the expression of pro-inflammatory cytokines such as TNF- $\alpha$, IL-1 $\beta$, IFN- $\gamma$, IL-12, cyclo-oxygenase (COX)-2 and inducible NO synthase ${ }^{(10,11)}$, and is required for proliferation/apoptosis homeostatic regulation and the protection from acute inflammation of the intestine ${ }^{(12,13)}$. Therefore, it is important to further elucidate the link between NF-кBdependent signalling pathways and dietary $n-3$ PUFA.

Curcumin is a natural polyphenol isolated from the dried rhizomes of Curcuma longa (turmeric). As the active component in turmeric, curcumin has been widely used in traditional medicine in India and Southeast Asia ${ }^{(14)}$. Moreover, curcumin has been shown to ameliorate inflammation associated with experimental colitis ${ }^{(15-17)}$. These data suggest that selective dietary polyphenolics may favourably modulate inflammatory responses in the colon. The chemoprotective effects of curcumin appear to be mediated, in part, through NF-кB inhibition $^{(17-19)}$. Therefore, in the present study, we have evaluated the effect of curcumin supplementation, in the presence or absence of dietary $n-3$ PUFA, on NF- $\mathrm{B}$ activation and the resolution of chronic inflammation in the colon.

Dextran sodium sulphate (DSS)-induced inflammation is an excellent preclinical mucosal wounding model of colitis that exhibits many phenotypic characteristics relevant to the human disease ${ }^{(20)}$. Typically, repeated cycles of DSS treatment are used to induce chronic intestinal inflammation ${ }^{(21)}$. In the present study, we exposed mice to either one or two cycles of DSS treatment to induce acute and chronic inflammation, respectively. We determined how dietary $n-3$ PUFA and curcumin influence (1) mortality, (2) colonic inflammation and injury scores, (3) epithelial cytokinetics, e.g. apoptosis and proliferation, (4) NF- $\mathrm{BB}$ activation and (5) global mucosal gene expression.

\section{Materials and methods}

\section{Animals and diet}

Male C57BL/6 mice, 6- to 8-weeks old, were obtained from Jackson Laboratories (Bar Harbor, ME, USA) and housed in a temperature- and humidity-controlled facility with a $12 \mathrm{~h}$ light $-12 \mathrm{~h}$ dark cycle. All procedures followed the guidelines approved by Public Health Service Policy and the Institutional Animal Care and Use Committee at Texas A\&M University. After 1 week of acclimatisation on a standard pelleted diet, animals were randomly grouped $(n 15)$ and fed ad libitum one of the four experimental diets $(2 \times 2$ design) (see Table S1 of the supplementary material, available online at http:// www.journals.cambridge.org/bjn): MO diet containing 5\%
MO; FO diet containing 4\% FO and 1\% MO (EPA and DHA content was 8.1 and $8.5 \mathrm{wt} \%$, respectively); MO plus curcumin diet (MO-cur) containing 5\% MO and $2 \%$ curcumin; FO plus curcumin diet (FO-cur) containing 4\% FO, 1\% MO and 2\% curcumin. The diets contained (per $100 \mathrm{~g}$ ) $42 \mathrm{~g}$ sucrose, $20 \mathrm{~g}$ casein, $22 \mathrm{~g}$ maize starch, $0.3 \mathrm{~g}$ DL-methionine, $3.5 \mathrm{~g} 76 \mathrm{~A}$ salt mix, $1.0 \mathrm{~g}$ American Institute of Nutrition (AIN) 76A mineral mix, $0.2 \mathrm{~g}$ choline chloride, $6 \mathrm{~g}$ fibre (cellulose), $5 \mathrm{~g}$ fat. The level of curcumin (2\%) has been shown to modulate disease pathology in the DSS mouse model ${ }^{(15)}$, and the relative dose is comparable with levels previously used in a human clinical trial $^{(22)}$. To prevent the formation of oxidised lipids, diets were stored at $-20^{\circ} \mathrm{C}$ and provided fresh to animals each day. $\mathrm{MO}$ was obtained from Dyets (Bethlehem, PA, USA), and vacuum deodorised menhaden FO was obtained from Omega Protein (Houston, TX, USA). Curcumin C3 complex, which contains $80 \%$ curcumin, 3\% bisdemethoxy curcumin and $17 \%$ demethoxy curcumin, was provided by Sabinsa Corporation (Payson, UT, USA). Heavy metals, including As and $\mathrm{Pb}$, were below the limits of detection.

\section{Colitis induction and histological scoring}

Animals were fed experimental diets 1 week before DSS (molecular weight, 36000-50000; MP Biomedicals, Solon, $\mathrm{OH}$, USA) treatment (see Fig. S1 of the supplementary material, available online at http://www.journals.cambridge.org/bjn). To induce chronic inflammation, $2.5 \%$ DSS was administered in the drinking-water for $5 \mathrm{~d}$, followed by $16 \mathrm{~d}$ of tap water. This cycle was repeated with a lower dose of DSS ( $3 \mathrm{~d}$ of $1.5 \%$ DSS followed by a $14 \mathrm{~d}$ recovery period) after which mice were euthanised humanely. Acute inflammation was induced by $5 \mathrm{~d}$ of $2.5 \%$ DSS, followed by a $3 \mathrm{~d}$ recovery period. At each necropsy interval, the entire colon was removed, flushed with PBS, fixed in $4 \%$ paraformaldehyde and paraffin embedded. Sections were stained with haematoxylin and eosin. Histological examination was performed in a blinded manner by a boardcertified pathologist (B. R. W.), and the degree of inflammation (score, 0-3) and epithelial injury (score, 0-3) in microscopic cross-sections of the colon was graded as described previously $^{(23)}$. Briefly, the presence of occasional inflammatory cells in the lamina propria was assigned a value of 0 ; increased numbers of inflammatory cells in the lamina propria as 1 ; confluence of inflammatory cells, extending into the submucosa, as 2; and transmural extension of the infiltrate as 3. For the evaluation of epithelial injury, no mucosal damage was scored as 0 ; discrete lympho-epithelial lesions were scored as 1 ; surface mucosal erosion or focal ulceration was scored as 2 and extensive mucosal damage associated with the deeper structures of the bowel wall was scored as 3 .

\section{In situ apoptosis and proliferation measurement}

Intestinal apoptosis was measured in paraformaldehyde-fixed, paraffin-embedded tissues using the terminal deoxynucleotidyl transferase-mediated uridine $5^{\prime}$ triphosphate-biotin nick-end (Oncor, Dallas, TX, USA) labelling method ${ }^{(24)}$. Cell proliferation was measured following bromodeoxyuridine 
injection (Zymed, South San Francisco, CA, USA) as described previously ${ }^{(25)}$.

\section{Assessment of NF- $\kappa B$ activity}

NF- $\kappa$ B activation was measured by quantifying p65/Rel A activation as described previously ${ }^{(26)}$. In brief, whole-cell protein from snap-frozen colonic mucosa scraping was extracted using a Nuclear Extraction Kit (Active Motif, Carlsbad, CA, USA) and subsequently incubated with oligonucleotides which comprise the NF- $\mathrm{KB}$ consensus DNA-binding site $\left(5^{\prime}\right.$-GGGACTTTCC-3') to detect activated p65/Rel A.

\section{Total RNA isolation}

At each necropsy interval, mucosa scrapings from the colon were stored in mirVana ${ }^{\mathrm{TM}}$ reagent at $-80^{\circ} \mathrm{C}$. Total RNA was extracted from each sample using mirVana ${ }^{\mathrm{TM}}$ miRNA Isolation Kit (Ambion, Austin, TX, USA) according to the manufacturer's instructions. The concentration and quality of total RNA were assessed using a Nanodrop spectrophotometer (Nanodrop, Wilmington, DE, USA) and an Agilent 2100 Bioanalyser (Agilent Technologies, Waldbronn, Germany), respectively.

\section{Codelink mouse whole-genome microarray assay}

Quadruplicate total RNA samples (including non-DSS, MO-fed control) were processed to generate biotin-labelled complementary RNA via a modified Eberwine RNA amplification protocol using a CodeLink iExpress Kit (Applied Microarray, Tempe, AZ, USA). Labelled complementary RNA was applied to CodeLink Mouse Whole Genome Bioarrays (Applied Microarray), which contain 34967 unique probe sequences. After incubation, slides were washed, stained and scanned by Gene Pix. Array images were processed using CodeLink system software version 5.0. The resulting files were imported into zRMicroArray ${ }^{(27)}$ for analysis. After median and log transformation, the gene expression was examined for normality (Shapiro-Wilk test) of the empirical distribution within each experimental group. Only genes that passed the significance test $(P<0.05)$ were considered for subsequent analyses ${ }^{(28)}$.

\section{Immunohistochemistry of pSTAT3}

Sections ( $4 \mu \mathrm{m}$ thick) were cut from paraffin-embedded colon 'swiss rolls' for immunohistochemistry of pSTAT3tyr705 (Cell Signalling, Danvers, MA, USA). In brief, tissue sections were deparaffinised and antigen retrieval was performed using EDTA buffer, pH 8. Primary antibody (1:50) was incubated overnight at $4^{\circ} \mathrm{C}$ followed by the addition of Signal Stain Boost IHC Detection Reagent (horseradish peroxidase, rabbit; Cell Signalling) for $30 \mathrm{~min}$. Slides were developed using diaminobenzidine. Staining of pSTAT3 was quantified using a Nikon Cool-SNAP camera and NIS elements software. From the distal, middle and proximal regions of the colon, four representative stained areas (hot spots) were selected, corresponding to a total of twelve images from each slide per animal. Stained areas in the mucosa within the intensity threshold were recorded. On average, twelve images per animal were read in a blinded fashion.

\section{Statistics}

Data are expressed as means with their standard errors. Differences between experimental groups were analysed using ANOVA from the SPSS software package and ANOVA R-package which is embedded in zRMicroArray. Adjusted $P$ values $<0.05$ were accepted as significant.

\section{Results}

\section{Body weight and colon length}

Animal body weight and colon length were recorded at termination points. Body weight dropped significantly following acute DSS treatment but no differences were observed between diets (see Fig. S2(A) of the supplementary material, available online at http://www.journals.cambridge.org/bjn). Similarly, colon length was shortened significantly following both acute and chronic DSS treatment, and no differences between diets were detected (see Fig. S2(B) of the supplementary material, available online at http://www.journals. cambridge.org/bjn).

\section{Dietary fish oil and curcumin, independently and in combination, enhance acute dextran sodium sulphate- induced mortality}

Animal mortality was determined following both acute (one cycle) and chronic (two cycles) DSS exposure. The MO diet was used as a baseline control because it contains no EPA, DHA or curcumin. Therefore, curcumin supplementation to the MO diet (MO-cur) represents the isolated effect of curcumin. The FO diet represents the effect of FO alone, while the FO-cur diet represents the combined effects of FO and curcumin. During the acute inflammatory phase (Fig. 1(A) and (C)), FO-fed mice exhibited the highest mortality (40\%, seventeen of forty-three) compared with MO with the lowest mortality ( $3 \%$, one of twenty-nine) $(P=0.0008)$. Addition of curcumin to MO increased $(P=0.003)$ the mortality to $37 \%$ (thirteen of thirty-five). FO and curcumin combination treatment also increased $(P=0.03)$ the mortality to $28 \%$. These results demonstrate that $\mathrm{FO}$ or curcumin supplementation increases the mortality rate following acute DSS exposure. Mice surviving acute colitis and exposed to a second round of DSS (chronic phase) died infrequently ( $\mathrm{MO}$, two of seventeen, FO, one of ten, MO-cur, one of eleven, FO-cur, two of fourteen) and exhibited no effect of diet (Fig. 1(B) and (D)). These data indicate that $n-3$ PUFA and curcumin supplementation exacerbate susceptibility to DSS-induced acute colitis.

Fish oil and curcumin modulate dextran sodium sulphateinduced pathology

To explore the effect of $n-3$ PUFA and curcumin on DSSinduced colitis, mice were treated with a single $5 \mathrm{~d}$ cycle of 

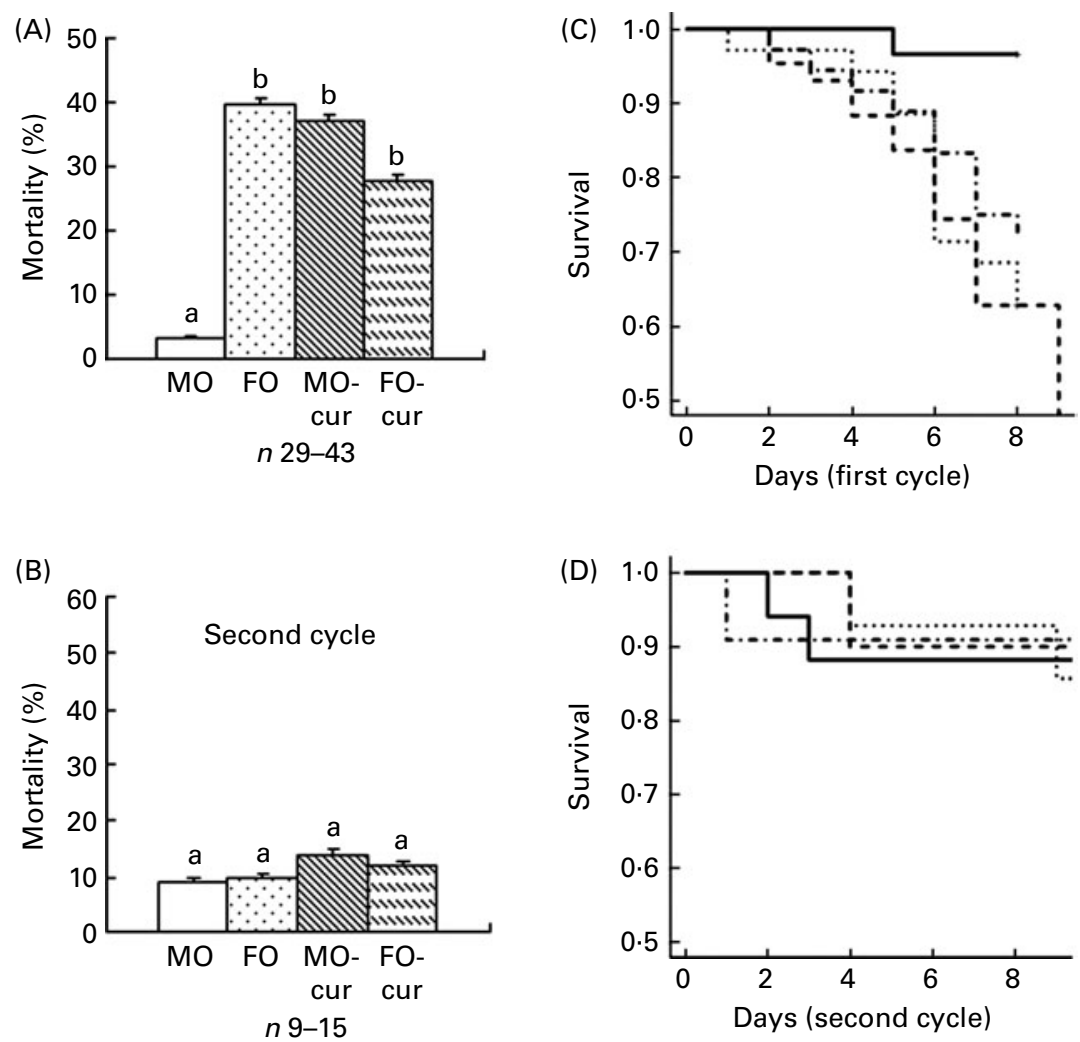

Fig. 1. Fish oil (FO) and curcumin feeding increase mortality. Animal mortality following dextran sodium sulphate (DSS) treatment was recorded during the (A) acute and (B) chronic inflammatory phases. Kaplan-Meier plots are shown for the (C) acute and (D) chronic experimental phases. Maize oil (MO, $\frac{}{-}$ ), $5 \%$ MO; FO (- - - ), $4 \%$ FO + 1\% MO; MO plus curcumin (MO-cur; -.........), $5 \% \mathrm{MO}+2 \%$ curcumin; FO plus curcumin (FO-cur; - - - ), $4 \%$ FO + $1 \% \mathrm{MO}+2 \%$ curcumin. Acute phase represents treatment with DSS for $5 \mathrm{~d}$ followed by a $3 \mathrm{~d}$ recovery period; chronic phase represents two cycles of DSS exposure followed by a $14 \mathrm{~d}$ recovery period. Refer to Fig. $\mathrm{S} 1$ of the supplementary material, available online at $\mathrm{http}: / / \mathrm{www}$.journals.cambridge.org/bjn for details. ${ }^{\mathrm{a}, \mathrm{b}} \mathrm{Mean}$ values with unlike letters were significantly different $(P<0.05)$.

DSS followed by a $3 \mathrm{~d}$ recovery (acute) or two cycles of DSS (chronic), and colonic inflammation and mucosal injury were assessed. Mouse colon sections were haematoxylin and eosin stained for histological scoring (Fig. 2(A)). Injury and inflammatory scores represent the degree of ulceration and immune cell infiltration, respectively. FO with or without curcumin supplementation and $\mathrm{MO}$ with curcumin increased $(P<0.05)$ injury scores, compared with control (MO), following both acute and chronic DSS exposure (Fig. 2(B)). This is consistent with animal survival data indicating that dietary FO and/or curcumin promoted mucosal injury and mortality during acute-phase DSS exposure (Fig. 1(A) and (C)). With respect to the inflammatory score, the addition of curcumin to FO was protective, especially following chronic DSS exposure (Fig. 2(C)). These data suggest that $n-3$ PUFA and curcumin may affect the pathology of DSS-induced colitis by modulating immune cell infiltration and activation in both acute and chronic phases.

\section{Fish oil and curcumin combination suppresses dextran} sodium sulphate-induced NF- $\kappa B$ activation

We examined NF- $\kappa$ B activation status following chronic DSS exposure in order to elucidate the mechanism by which $n$-3 PUFA and curcumin modulate the inflammatory response in the colon. In comparison with the MO control, only the dietary FO and curcumin combination suppressed NF-кB activity in the colonic mucosa (Fig. 3). Together with inflammatory score data (Fig. 2), these results suggest a mechanistic link between the enhanced resolution of chronic inflammation and the suppression of NF-кB by FO-cur feeding.

\section{Fish oil and curcumin combination promotes mucosal cell proliferation in the colon}

Next, we investigated the ability of FO and curcumin to modulate mucosal cytokinetics following DSS-induced exposure. Epithelial apoptosis was measured by the terminal deoxynucleotidyl transferase (terminal deoxynucleotidyl transferasemediated uridine 5 triphosphate-biotin nick-end labelling) assay. As shown in Fig. 4(A) and Fig. S3 of the supplementary material (available online at http://www.journals.cambridge. org/bjn), dietary FO enhanced epithelial apoptosis compared with the MO control, which may explain, in part, the elevated injury score associated with FO feeding (Fig. 2(B)). Since the initiation of cell proliferation is a prerequisite for mucosal repair $^{(29)}$, we examined epithelial cell proliferation by bromodeoxyuridine incorporation. As shown in Fig. 4(B), feeding FO-cur resulted in an enhanced rate of proliferation relative 
(A)

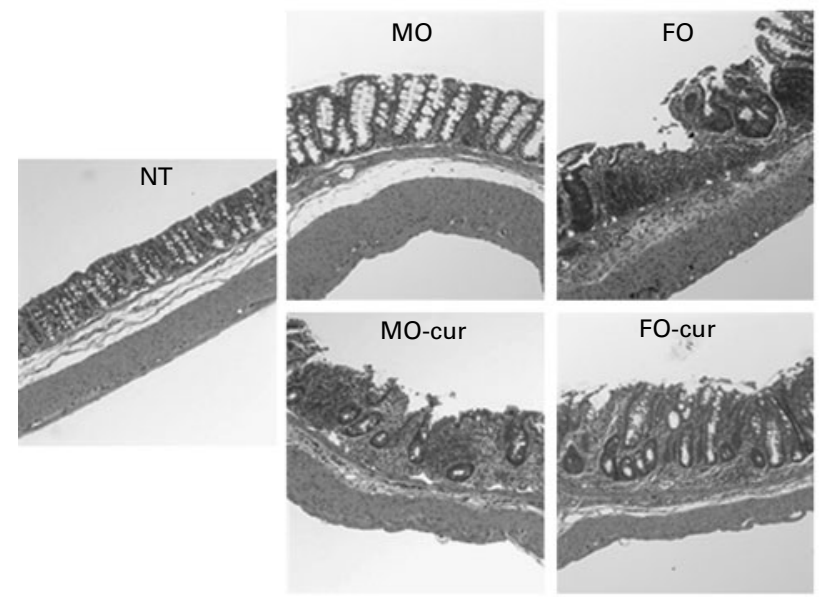

(B)
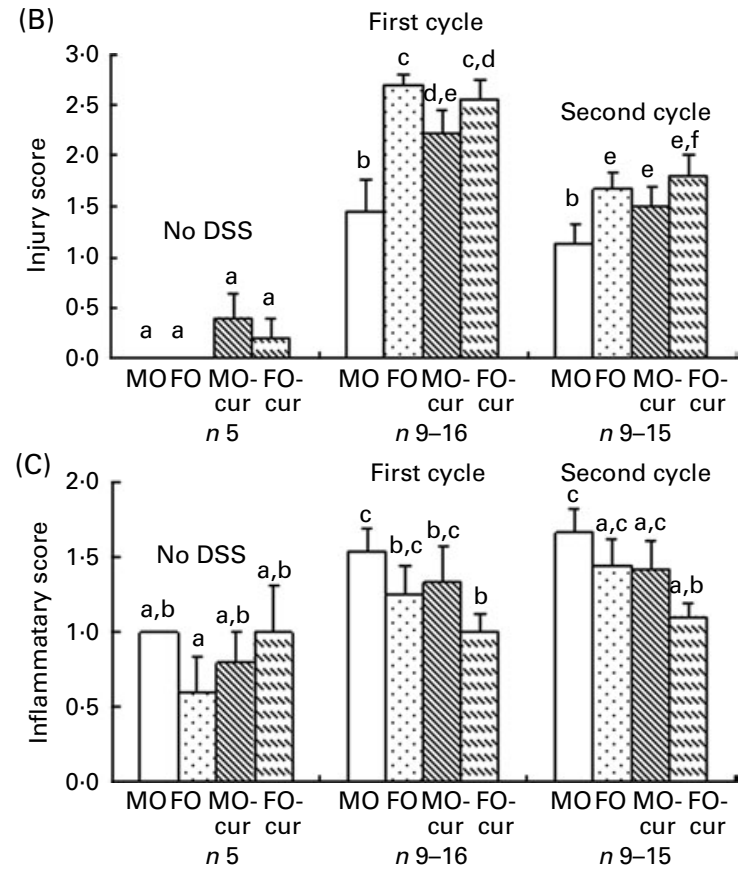

Fig. 2. Histological features of colonic inflammation and mucosal injury. Mice were treated with an acute or chronic dextran sodium sulphate (DSS) regimen, and colonic inflammation and mucosal injury were assessed. (A) Representative haematoxylin and eosin-stained colonic sections from mice were exposed to chronic DSS treatment (100 $\times$ magnification). Crypts were severely distorted and the epithelium was denuded in fish oil (FO), FO curcumin and maize oil (MO) curcumin treatments compared with MO (control) and non-DSS-treated animals fed MO (NT). (B) Injury scores and (C) inflammatory scores. Values are means, with their standard errors represented by vertical bars. No DSS, indicates no DSS treatment; first cycle, indicates $5 \mathrm{~d}$ DSS followed by a $3 \mathrm{~d}$ recovery period; second cycle, indicates two cycles of DSS exposure. ${ }^{\mathrm{a}, \mathrm{b}, \mathrm{c}, \mathrm{d}, \mathrm{e}, \mathrm{f}}$ Mean values with unlike letters were significantly different $(P<0.05)$. MO-cur, MO plus curcumin; FO-cur, FO plus curcumin.

to $\mathrm{MO}$ (control). This suggests that the FO-cur diet promotes repair of the colonic epithelium.

\section{Diet and dextran sodium sulphate exposure modulate mucosal gene expression}

Gene expression in chronic colitis was determined by comparing the treated groups (two cycles of DSS) with MO-fed no-DSS untreated control (MO-con) mice. As shown in Fig. 5, genes up- or down-regulated by FO, curcumin and FO-cur combination were contrasted in order to elucidate the biological processes contributing to the tissue injury and inflammation phenotypes. Because dietary FO-cur reduced the inflammatory score and NF- $\mathrm{KB}$ activation, and elevated intestinal cell proliferation (Figs. 2-4), we compared gene expression profiles from mice fed FO-cur with the mice fed the MO control diet without DSS treatment (MO-con) (Fig. 5(A)). Specifically, genes that were differentially expressed in the MO, MO-cur and $\mathrm{FO}$ groups relative to the MO-con following chronic colitis but whose change was blocked by the FO-cur diet were identified. Examples include a hypoxic perinecrotic marker of tumour angiogenesis, neuritin precursor $(\mathrm{Nrn} 1)^{(30)}$, which was down-regulated by 0.4-0.5-fold in the MO-DSS, FO-DSS, MO-cur-DSS groups relative to the $\mathrm{MO}-\mathrm{con}$ and $\mathrm{FO}-\mathrm{cur}-\mathrm{DSS}$ treatment. In addition, the FO-cur combination prevented the up-regulation of pro-inflammatory genes (e.g. lymphocyte antigen 9 (Slamf3), suppressor of cytokine signalling 3 (Socs3), TNF receptor superfamily member 1B precursor (Tnfrsf1b) and macrophage colony-stimulating factor 1 receptor precursor (Csf1r)), expressed in immune cells ${ }^{(31-33)}$. These observations are consistent with the ability of FO-cur to resolve inflammation (Fig. 2). Up-regulation of the secretory phospholipase $A_{2}$ precursor (Pla2g2c) and myosin regulatory light chain 2 (Myl7) was also prevented in the FO-cur-DSS group.

Genes that were affected by curcumin alone are shown in Fig. 5(B). In this comparison, diets containing curcumin

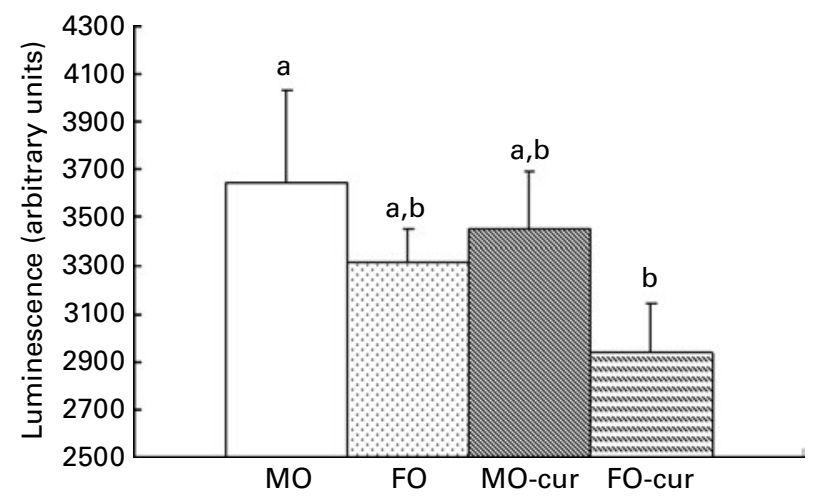

Fig. 3. Fish oil (FO) and curcumin suppress NF-кB activation in the colonic mucosa. Colonic mucosa was isolated following two cycles of dextran sodium sulphate treatment (chronic inflammatory phase) and p65 NF-кB nuclear activation was determined. Values are means, with their standard errors represented by vertical bars (seven mice per treatment). ${ }^{a, b}$ Mean values with unlike letters were significantly different $(P<0.05)$. Refer to Fig. 1 for legend details. MO-cur, maize oil plus curcumin; FO-cur, FO plus curcumin. 

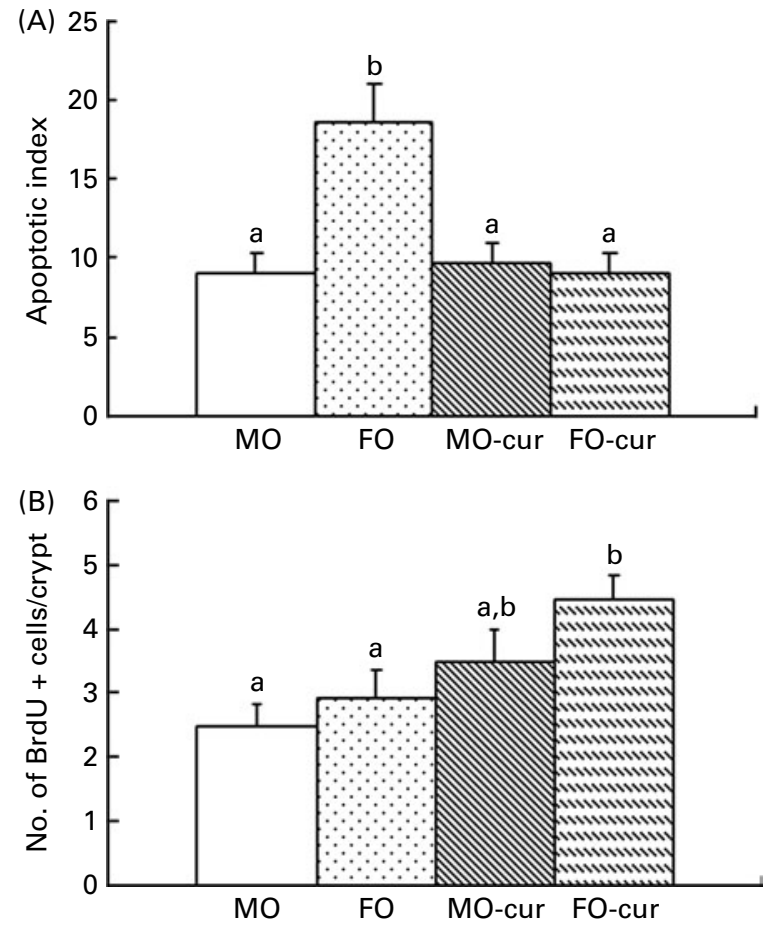

Fig. 4. Fish oil (FO) and curcumin modulate colonocyte cytokinetics. In situ detection of $(A)$ epithelial apoptosis and $(B)$ proliferation was quantified using terminal deoxynucleotidyl transferase-mediated uridine 5 triphosphate-biotin nick-end labelling and bromodeoxyuridine (BrdU) incorporation, respectively. Animals were terminated following two cycles of dextran sodium sulphate treatment (chronic inflammatory phase). Data are expressed as an apoptotic index (total number of apoptotic cells per 100 crypts). Values are means, with their standard errors represented by vertical bars (three to five mice per treatment, $115-338$ crypts per column). ${ }^{a, b}$ Mean values with unlike letters were significantly different $(P<0.05)$. MO-cur, maize oil plus curcumin; FO-cur, FO plus curcumin.

(MO-cur-DSS and FO-cur-DSS) reduced the expression of ELKS/RAB6-interacting/CAST family member 1 (Erc1) during chronic inflammation to levels similar to the MO-con diet. Erc1 is an essential regulatory subunit of the IкB kinase complex and important for NF- $\mathrm{KB}$ activation ${ }^{(34)}$, suggesting that curcumin is capable of modulating chronic inflammation via the inhibition of NF- $\mathrm{BB}$ activation in the colon mucosa. Curcumin also blocked the DSS-induced increase of proinflammatory genes such as T-cell surface glycoprotein CD5 precursor (Cd5), paired Ig-like receptor B (Lilrb3), cAMPspecific $3^{\prime}, 5^{\prime}$-cyclic phosphodiesterase 4B (Pde4b), nuclear body protein (Sp110) and solute carrier family 35, member E3 (Slc35e3). It is noteworthy that Pde4b inhibitors exhibit profound anti-inflammatory effects and have utility in the treatment of cancer and the regulation of energy balance ${ }^{(35,36)}$.

Genes that were selectively affected by FO feeding are shown in Fig. 5(C). In this comparison, mice that received FO-DSS and FO-cur-DSS treatment produced an expression profile similar to MO-con. Of interest in Fig. 5(C), two NF-кB-regulated genes had reduced the expression in response to $\mathrm{FO}$, i.e. Toll-like receptor 12 (Tlr12) and signal transducer and activator of transcription 3 (Stat3). An immunohistochemical analysis of pSTAT3 Tyr 705 performed to confirm the microarray results showed that FO-DSS and
FO-cur-DSS treatment had suppressed levels of pSTAT3 compared with MO-cur-DSS treatment but not MO-DSS (see Fig. S4 of the supplementary material, available online at http:// www.journals.cambridge.org/bjn). Although the function of Tlr12 in colonic inflammation is not known, Stat 3 has been reported to be a required regulator for the survival of intestinal epithelial cells and the development of colitis-associated tumorigenesis ${ }^{(37,38)}$. FO feeding also reduced the expression of pro-inflammatory genes including low-affinity Ig $\gamma \mathrm{FC}$ region receptor III precursor (Fcgr3), carcinoembryonic antigen-related cell adhesion molecule 14 (Ceacam14), early T-cell activation antigen p60 (Cd69), TNF $\alpha$-induced protein 2 (Tnfaip2) and basic leucine zipper transcriptional factor ATF-like 3 (Batf3). Interestingly, Batf3 may be required for the differentiation of IL17-producing T-helper (TH17) cells ${ }^{(39)}$.

We also examined genes that were uniquely affected by either FO or curcumin treatment, i.e. changes that were exclusive to MO-DSS treatment (Fig. 5(D)). Using this comparison, two NF- $\mathrm{B}$ target genes, transforming growth factor $\beta-3$ precursor (Tgfb3) and AP-1 complex subunit sigma-2 (Ap1s2), were selectively up-regulated in MO-DSS mice. This is noteworthy because TGF $\beta 3$ is a well-known pro-inflammatory marker of $\mathrm{IBD}^{(40)}$. Other effector pro-inflammatory genes which were up-regulated include scavenger receptor class A member 3 (Scara3), paired Ig-like type 2 receptor $\beta 2$ precursor (Pilrb2), killer cell lectin-like receptor, subfamily A, member 17 (Klra17) $^{(41-43)}$, Ig heavy-chain C gene segment (Igh) and histidine decarboxylase ( $\mathrm{Hdc}$ ). Interestingly, $\mathrm{Hdc}$ is modulated by TLR agonists ${ }^{(44)}$. MO plus DSS treatment also increased additional genes that may be involved in mucosa wound repair including pro-neuregulin-1 $(\mathrm{Nrg} 1)^{(45)}$, chondroitin sulphate synthase 1 (Chsy1) ${ }^{(46)}$ and epidermal growth factor receptor kinase substrate 8-like protein 1 (Eps811) $^{(47)}$. Genes specifically down-regulated by MO feeding in the presence of DSS include elongation factor Tu, mitochondrial precursor (Tufm), nicotinate phosphoribosyltransferase (Naprt1), cytochrome $\mathrm{P} 450$, family 4, subfamily $\mathrm{f}$, polypeptide 13 (Cyp4f13) and Zn finger CCCH domain-containing protein 8 (Zc3h8).

\section{Discussion}

Dietary $n-3$ PUFA and curcumin are receiving substantial attention for their anti-tumorigenic and anti-inflammatory properties $^{(23,48-50)}$. In the present study, we assessed the combined effects of these two bioactive dietary components on the resolution of both acute and chronic experimental colitis. FO and curcumin, both alone and in combination, unexpectedly enhanced animal mortality and exacerbated colonic mucosa injury following an acute inflammatory episode (Figs. 1(A), (C) and Fig. 2(B)). Wound repair occurs in three overlapping but distinct stages: inflammation; new tissue formation; remodelling ${ }^{(51)}$. Inflammation occurs immediately after tissue damage and is essential for wound repair. In refractory cases of IBD or experimental chronic inflammation, mucosal repair is disrupted. Repair of damaged intestinal mucosa is regulated by multiple factors at both molecular and cellular levels including innate and adaptive immune 
(A)

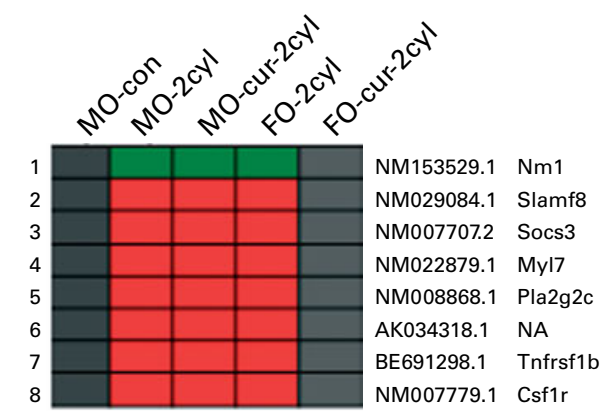

(C)

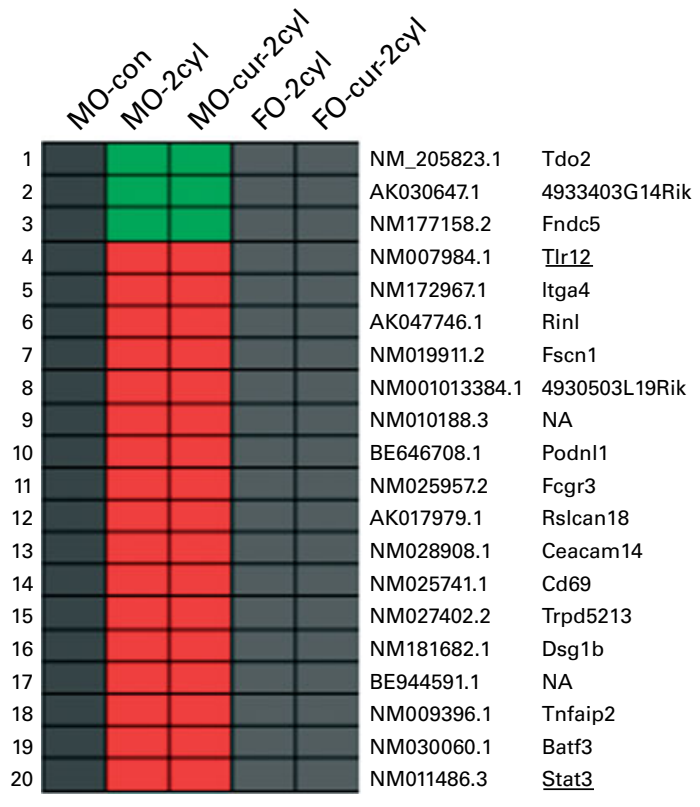

(B)

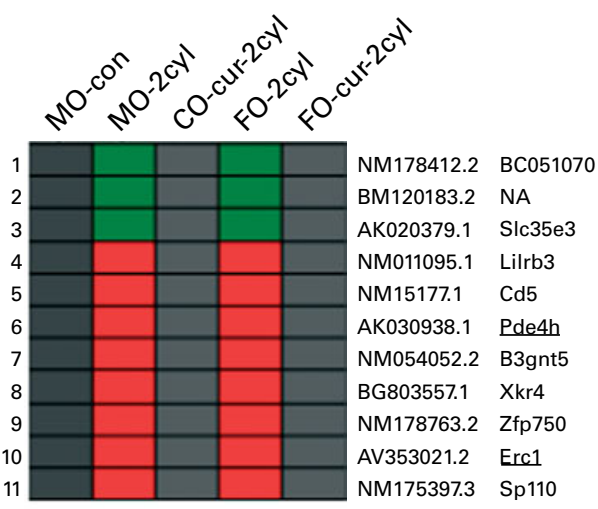

(D)

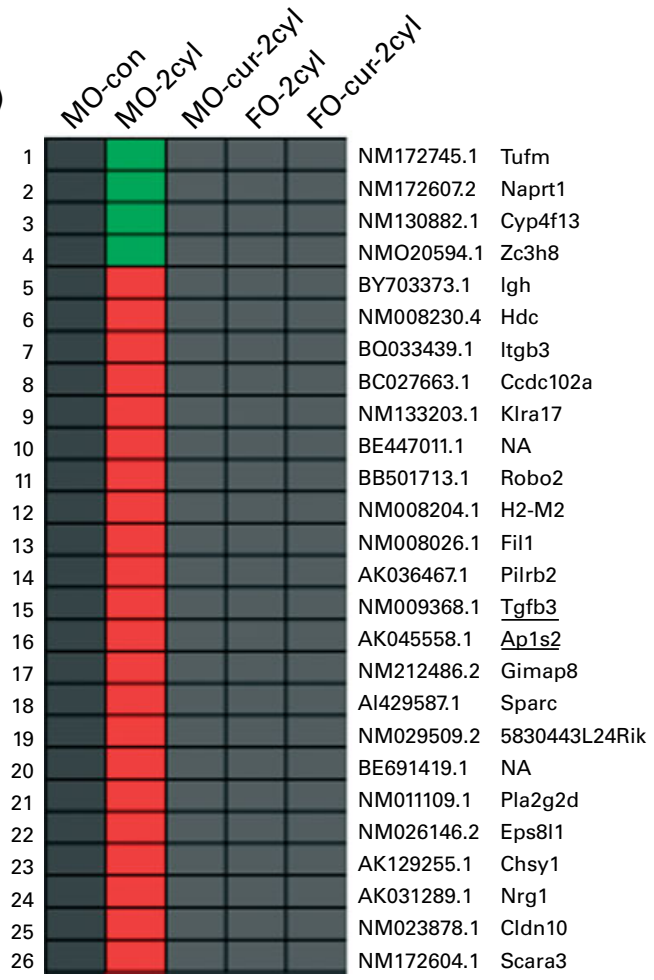

Fig. 5. Fish oil (FO) and curcumin supplementation differentially regulate intestinal gene expression. Distinctive gene expression signatures are found in the colonic mucosa following FO plus curcumin (FO-cur) dietary treatments and exposure to dextran sodium sulphate (DSS). Functional annotation of gene sets with similar profiles are compared: (A) expression pattern for genes strictly affected by FO-cur combination therapy; i.e. no DSS treatment maize oil (MO) control (con) diet (MO-con) and FO-cur-DSS combination $v$. other, (B) expression pattern for genes strictly affected by curcumin therapy; i.e. MO-con, MO plus curcumin (MO-cur)-DSS, and FO-cur-DSS supplementation $v$. other, (C) expression pattern for genes strictly affected by FO feeding; i.e. MO-con, FO-DSS, FO-cur-DSS $v$. other, (D) expression pattern for genes affected by FO or curcumin treatment; i.e. MO-DSS effect, MO-DSS (positive con) $v$. other. Significantly $(P<0.05)$ up-regulated genes are shown in red, and down-regulated genes are shown in green. Black boxes indicate no significant difference $(P>0.05)$ compared with MO-con. NF-kB-related genes are underlined. $n 5$ Mice for MO-con, $n$ 9-10 for all other groups. All animals were exposed to two DSS cycles (cyl) except for MO-con. NA, gene name not available.

cells ${ }^{(29,52,53)}$. Although many elements of the acute inflammatory response influence wound repair, particular attention has been paid to growth factors, PG and cytokines ${ }^{(53,54)}$. Interestingly, there is a growing body of evidence indicating that $n-3$ PUFA $^{(48,55)}$ and curcumin ${ }^{(26,56,57)}$ inhibit these same mediators, which are required for protection from DSS-induced colonic injury. Although there is a significant redundancy in the inflammatory response ${ }^{(51)}$, it is possible that $\mathrm{FO}$ and curcumin delay epithelial repair mechanisms, which would enhance absorption of bacterial toxins and, therefore, increase mortality. There is also a remote possibility that dietary lipid source may influence curcumin bioavailability. However, there is no precedent in the literature to suggest that the lipid source can influence curcumin absorption/metabolism/ bioactivity.

The second stage of repair of injured intestinal mucosa, new tissue formation, is a process of organised restitution, proliferation and differentiation of mucosal epithelium. Within hours of injury, re-epithelialisation is initiated and epithelial cell proliferation is stimulated in crypts near the damaged mucosal area ${ }^{(29,52)}$. Our data show that FO-cur enhanced colonic epithelial proliferation compared with other diets (Fig. 4(B)), 
which suggests that this nutritional combination may promote mucosal repair during chronic inflammation. In contrast, FO alone increased epithelial cell apoptosis (Fig. 4(A)). This is noteworthy because sustained epithelial apoptosis can preclude mucosal healing and decrease animal survival ${ }^{(4)}$, which may explain the elevated injury scores observed in FO-fed mice during acute-phase inflammation. Along these lines, it has been shown that the balance between colonic epithelial cell proliferation and apoptosis can be modulated by dietary $n$-3 PUFA, conferring resistance to toxic carcinogenic agents $^{(4,25,58)}$. Additional experiments are required to further address the impact of diet on colonic cytokinetics following acute DSS exposure.

With respect to mechanisms which mediate intestinal cell cytokinetics, it has been shown that Toll-like receptor-4 (TLR4)-PG-dependent signalling directly regulates proliferation and apoptosis in response to acute colitis ${ }^{(54,59,60)}$. Although TLR4 activation is beneficial in the short term, chronic signalling may lower the threshold for inflammationassociated colon cancer ${ }^{(61)}$. With respect to diet, DHA and curcumin appear to be pan-inhibitors for various $\operatorname{TLR}^{(62,63)}$ Additional studies are needed in order to determine if these observations can be validated in vivo. It is also well documented that $n-3$ PUFA (EPA and DHA) antagonise arachidonic acid-derived $\mathrm{PG}\left(\mathrm{PGE}_{2}\right.$ and $\left.\mathrm{PGD}_{2}\right)$ in the colonic mucosa ${ }^{(24,64)}$. Curcumin has also been reported to suppress $\mathrm{PGE}_{2}$ formation by blocking the expression of COX-2 and microsomal $\mathrm{PGE}_{2}$ synthase-1 and inhibiting the activity of microsomal $\mathrm{PGE}_{2}$ synthase- ${ }^{(56)}$. This is noteworthy because $\mathrm{PGE}_{2}$ is capable of enhancing cell proliferation, angiogenesis, cell migration and invasion as well as inhibiting apoptosis and enhancing tumour growth ${ }^{(65)}$. Recently, it has been demonstrated that $\mathrm{PGE}_{2}$ is important for the healing of ulcers and epithelial injury ${ }^{(66,67)}$. Taken together, these data suggest that $n-3$ PUFA and curcumin modulate the resolution of inflammation and mucosa repair, in part, by suppressing the TLR4/COX-2/ $\mathrm{PGE}_{2}$ signalling axis.

We demonstrate for the first time that FO with or without curcumin exacerbates acute inflammatory responses in the DSS wounding mouse model. Specifically, MO (control diet)fed, DSS-treated mice were protected against acute death and mucosal damage compared with the FO with or without curcumin treatments (Figs 1 and 2). In contrast, FO-cur afforded the greatest protection with respect to mucosal (acute and chronic) inflammation (Fig. 2). Overall, these data indicate an enhanced ability of FO-cur-fed mice to resolve chronic inflammation of the colon. From a mechanistic standpoint, dietary FO-cur combination significantly suppressed NF- $\mathrm{BB}$ activity in the colon (Figs. 2(C) and 3), suggesting that $\mathrm{FO}$ and curcumin act in a combinatory manner. The separate effects of these two bioactive components have been reported previously ${ }^{(9,18,68)}$. NF- $\kappa \mathrm{B}$ is an important intracellular regulator of both the inflammatory response and mucosal integrity via the TLR4/COX-2/PGE2 signalling axis, which is important for the healing of epithelial injury $^{(67)}$. Recent findings have indicated that NF-кB can exert either a deleterious or a protective function in the intestine, depending on the stimuli encountered ${ }^{(11-13,48)}$. NF-кB regulates the gene expression of pro-inflammatory mediators including IL-1 $\beta$, TNF- $\alpha$, IL-12p40 and IL-23p19, which contribute to the pathophysiology of chronic intestinal inflammatory diseases $^{(11-13)}$.

Mucosal microarray analysis revealed that dietary FO, curcumin and FO-cur combination differentially modulated the expression of genes induced by DSS treatment (Fig. 5). A regulator of NF- $\mathrm{BB}$ activation, Erc1, was suppressed at the transcriptional level following supplementation with curcumin (Fig. 5(B)). As an essential regulatory subunit of the IкB kinase (IKK) complex ${ }^{(34)}$, Erc1 may be a new target for curcumin which modulates NF- $\mathrm{BB}$ activation in addition to inhibiting IKK and Akt activation ${ }^{(69)}$. The suppression of $\mathrm{NF}-\kappa \mathrm{B}$ target genes by FO or curcumin is consistent with the critical role of this transcription factor as a key regulator of intestinal inflammation. Interestingly, FO feeding blocked DSS-induced Stat3 up-regulation (Fig. 5(C)). Stat3 has been reported recently as a key regulator in IBD and colon cancer ${ }^{(37,38)}$. Specifically, Stat3 activation in enterocytes is required for cell survival and its hyperactivation promotes colitis associated tumorigenesis and growth ${ }^{(37,38)}$. In addition to intestinal epithelial cells, Stat3 is constitutively activated in mucosa immune cell types including dendritic cells, macrophages and T-cells ${ }^{(70-72)}$. The Stat 3 pathway in $\mathrm{CD}^{+}{ }^{+} \mathrm{T}$ cells promotes IL-17-producing Th cell (Th17) development which mediates immune responses in autoimmune disease and IBD-induced cancer development ${ }^{(70,71)}$. Stat 3 activation in immune cells promotes an IL-23-mediated procarcinogenic immune response while inhibiting IL-12-dependent Th1-mediated antitumour immunity ${ }^{(72)}$. Our results suggest that Stat 3 may be a new target of the antitumorigenic $n-3$ PUFA. We are currently investigating whether $n-3$ PUFA modulate Th17 polarisation in the intestine. Overall, FO and curcumin suppressed the up-regulation of pro-inflammatory gene expression relative to the MO (control) diet, which is consistent with human studies showing that EPA + DHA intake decreases the gene expression of inflammatory pathways, including NF-кB signalling ${ }^{(50,73,74)}$.

It has been reported that different strains of mice may have differential susceptibility to, and pathogenesis of, DSS-induced colitis and colon cancer ${ }^{(75-77)}$. DSS-induced chronic colitis in $\mathrm{C} 57 \mathrm{BL} / 6$ mice is believed to be a robust model for validating future therapies for the treatment of IBD because of the similarities of the pathology compared with symptoms in human disease $^{(77)}$. Thus, although different results might be obtained with another mouse strain, we believe that our findings make an important contribution to the consideration of using FO and curcumin as therapeutic agents in chronic IBD. We propose that $n-3$ PUFA and curcumin may be best suited to maintain IBD remission ${ }^{(78)}$.

In conclusion, our data show that dietary FO and curcumin differentially modulate the pathology of DSS-induced chronic colitis in mice. Feeding FO-cur together enhanced the resolution of chronic inflammation but disrupted mucosa repair during the acute phase of DSS exposure in part due to the inhibition of NF- $\mathrm{KB}$ activity in the colonic mucosa. FO and curcumin also differentially modulated mucosa cytokinetics, which may be attributed to the regulation of the 
TLR4/COX-2/PGE 2 and Stat3 signalling pathways in the colon. Collectively, these findings contribute to a better understanding of the ability of $n-3$ PUFA and curcumin to modulate the inflammation-mucosa repair-carcinogenesis axis in the colon.

\section{Acknowledgements}

The present study was financially supported by NIH grants DK071707, CA59034, CA129444 and USDA 2008-34402-19195 Vegetable \& Fruit Improvement Center. Curcumin C3 complex was provided by Sabinsa Corporation. Q. J. performed the experiments, data collection, analysis and interpretation of the data. I. I. and Z. Z. Z. performed the microarray data analysis. R. C. A., J. R. L., D. N. M. and R. S. C. assisted with the study design and data interpretation. B. R. W. scored the histology sections. E. S. C., J. S. G., L. A. D. and Y.-Y. F. contributed to the sample collection/reagents/materials and the development of analytical tools. L. Z. supervised the statistical analyses. None of the authors has a conflict of interest.

\section{References}

1. Mudter J \& Neurath MF (2003) Mucosal T cells: mediators or guardians of inflammatory bowel disease? Curr Opin Gastroenterol 19, 343-349.

2. Yamamoto-Furusho JK \& Podolsky DK (2007) Innate immunity in inflammatory bowel disease. World J Gastroenterol 13, 5577-5580.

3. Rubin DT \& Kavitt RT (2006) Surveillance for cancer and dysplasia in inflammatory bowel disease. Gastroenterol Clin North Am 35, 581-604.

4. Davidson LA, Nguyen DV, Hokanson RM, et al. (2004) Chemopreventive $n-3$ polyunsaturated fatty acids reprogram genetic signatures during colon cancer initiation and progression in the rat. Cancer Res 64, 6797-6804.

5. Prescott SM \& Stenson WF (2005) Fish oil fix. Nature Med 11, 596-598.

6. Hudert CA, Weylandt KH, Lu Y, et al. (2006) Transgenic mice rich in endogenous omega-3 fatty acids are protected from colitis. Proc Natl Acad Sci U S A 103, 11276-11281.

7. Chang WC, Chapkin RS \& Lupton JR (1997) Predictive value of proliferation, differentiation and apoptosis as intermediate markers for colon tumorigenesis. Carcinogenesis $\mathbf{1 8}$, $721-730$.

8. Whelan J \& McEntee MF (2004) Dietary (n-6) PUFA and intestinal tumorigenesis. J Nutr 134, 3421S-3426S.

9. Nowak J, Weylandt KH, Habbel P, et al. (2007) Colitis-associated colon tumorigenesis is suppressed in transgenic mice rich in endogenous $n-3$ fatty acids. Carcinogenesis $\mathbf{2 8}$, 1991-1995.

10. Hollenbach E, Vieth M, Roessner A, et al. (2005) Inhibition of RICK/nuclear factor-kappaB and p38 signaling attenuates the inflammatory response in a murine model of Crohn's disease. J Biol Chem 280, 14981-14988.

11. Waetzig GH, Seegert D, Rosenstiel P, et al. (2002) p38 Mitogen-activated protein kinase is activated and linked to TNF-alpha signaling in inflammatory bowel disease. J Immunol 168, 5342-5351.

12. Karrasch T \& Jobin C (2008) NF-kappaB and the intestine: friend or foe? Inflamm Bowel Dis 14, 114-124.

13. Steinbrecher KA, Harmel-Laws E, Sitcheran R, et al. (2008) Loss of epithelial RelA results in deregulated intestinal proliferative/apoptotic homeostasis and susceptibility to inflammation. J Immunol 180, 2588-2599.

14. Duvoix A, Blasius R, Delhalle S, et al. (2005) Chemopreventive and therapeutic effects of curcumin. Cancer Lett $\mathbf{2 2 3}$, 181-190.

15. Deguchi Y, Andoh A, Inatomi O, et al. (2007) Curcumin prevents the development of dextran sulfate sodium (DSS)induced experimental colitis. Dig Dis Sci 52, 2993-2938.

16. Jian YT, Mai GF, Wang JD, et al. (2005) Preventive and therapeutic effects of NF-kappaB inhibitor curcumin in rats colitis induced by trinitrobenzene sulfonic acid. World J Gastroenterol 11, 1747-1752.

17. Camacho-Barquero L, Villegas I, Sánchez-Calvo JM, et al. (2007) Curcumin, a Curcuma longa constituent, acts on MAPK p38 pathway modulating COX-2 and iNOS expression in chronic experimental colitis. Int Immunopharmacol 7 , 333-342.

18. Bharti AC, Donato N, Singh S, et al. (2003) Curcumin (diferuloylmethane) down-regulates the constitutive activation of nuclear factor-kappa B and IkappaBalpha kinase in human multiple myeloma cells, leading to suppression of proliferation and induction of apoptosis. Blood 101, 1053-1062.

19. Milacic V, Banerjee S, Landis-Piwowar KR, et al. (2008) Curcumin inhibits the proteasome activity in human colon cancer cells in vitro and in vivo. Cancer Res 68, 7283-7292.

20. Seril DN, Liao J, Yang GY, et al. (2003) Oxidative stress and ulcerative colitis-associated carcinogenesis: studies in humans and animal models. Carcinogenesis 24, 3533-3562.

21. Xiao H, Gulen MF, Qin J, et al. (2007) The Toll-interleukin-1 receptor member SIGIRR regulates colonic epithelial homeostasis, inflammation, and tumorigenesis. Immunity $\mathbf{2 6}$, $461-475$.

22. Hanai H \& Sugimoto K (2009) Curcumin has bright prospects for the treatment of inflammatory bowel disease. Curr Pharm Des 15, 2087-2094.

23. Jia Q, Lupton JR, Smith R, et al. (2008) Reduced colitisassociated colon cancer in fat- 1 ( $n-3$ fatty acid desaturase) transgenic mice. Cancer Res 68, 3985-3991.

24. Hong MY, Bancroft LK, Turner ND, et al. (2005) Fish oil decreases oxidative DNA damage by enhancing apoptosis in rat colon. Nutr Cancer 52, 166-175.

25. Ma DW, Finnell RH, Davidson LA, et al. (2005) Folate transport gene inactivation in mice increases sensitivity to colon carcinogenesis. Cancer Res 65, 887-897.

26. Kim W, Fan YY, Smith R, et al. (2009) Dietary curcumin and limonin suppress $\mathrm{CD}^{+}{ }^{+}$T-cell proliferation and interleukin-2 production in mice. J Nutr 139, 1042-1048.

27. Zlatev ZZ (2009) zRMicroArray: a computational environment for analyses of gene expression. Masters Thesis, Sofia University, Bulgaria.

28. Strimmer K (2008) A unified approach to false discovery rate estimation. BMC Bioinformatics 9, 303.

29. Okamoto R \& Watanabe M (2005) Cellular and molecular mechanisms of the epithelial repair in IBD. Dig Dis Sci $\mathbf{5 0}$, Suppl. 1, S34-S38.

30. Le Jan S, Le Meur N, Cazes A, et al. (2006) Characterization of the expression of the hypoxia-induced genes neuritin, TXNIP and IGFBP3 in cancer. FEBS Lett 580, 3395-3400.

31. Kingsbury GA, Feeney LA, Nong Y, et al. (2001) Cloning, expression, and function of BLAME, a novel member of the CD2 family. J Immunol 166, 5675-5680.

32. Baker BJ, Akhtar LN \& Benveniste EN (2009) SOCS1 and SOCS3 in the control of CNS immunity. Trends Immunol 30, 392-400. 
33. Patel S \& Player MR (2009) Colony-stimulating factor-1 receptor inhibitors for the treatment of cancer and inflammatory disease. Curr Top Med Chem 9, 599-610.

34. Ducut Sigala JL, Bottero V, Young DB, et al. (2004) Activation of transcription factor NF-kappaB requires ELKS, an IkappaB kinase regulatory subunit. Science 304, 1963-1967.

35. Smith PG, Wilkinson KN, Savage KJ, et al. (2005) The phosphodiesterase PDE4B limits cAMP-associated PI3K/ AKT-dependent apoptosis in diffuse B-cell lymphoma. Blood 105, 308-316.

36. Zhang R, Maratos-Flier E \& Flier JS (2009) Reduced adiposity and high-fat diet-induced adipose inflammation in mice deficient for phosphodiesterase 4B. Endocrinology 150, 3076-3082

37. Bollrath J, Phesse TJ, von Burstin VA, et al. (2009) gp130Mediated Stat 3 activation in enterocytes regulates cell survival and cell-cycle progression during colitis-associated tumorigenesis. Cancer Cell 15, 91-102.

38. Grivennikov S, Karin E, Terzic J, et al. (2009) IL-6 and Stat3 are required for survival of intestinal epithelial cells and development of colitis-associated cancer. Cancer Cell 15, $103-113$

39. Schraml BU, Hildner K, Ise W, et al. (2009) The AP-1 transcription factor Batf controls $\mathrm{T}(\mathrm{H}) 17$ differentiation. Nature 460, 405-409.

40. Kanazawa S, Tsunoda T, Onuma E, et al. (2001) VEGF, basicFGF, and TGF-beta in Crohn's disease and ulcerative colitis: a novel mechanism of chronic intestinal inflammation. $A m J$ Gastroenterol 96, 822-828.

41. Balla B, Kósa JP, Kiss J, et al. (2009) Transcriptional profiling of immune system-related genes in postmenopausal osteoporotic versus non-osteoporotic human bone tissue. Clin Immunol 131, 354-359.

42. Wilson MD, Cheung J, Martindale DW, et al. (2006) Comparative analysis of the paired immunoglobulin-like receptor (PILR) locus in six mammalian genomes: duplication, conversion, and the birth of new genes. Physiol Genomics 27, 201-218.

43. Tai LH, Goulet ML, Belanger S, et al. (2008) Positive regulation of plasmacytoid dendritic cell function via Ly49Q recognition of class I MHC. I Exp Med 205 , 3187-3199.

44. Funayama H, Huang L, Asada Y, et al. (2010) Enhanced induction of a histamine-forming enzyme, histidine hecarboxylase, in mice primed with nod1 or nod2 ligand in response to various toll-like receptor agonists. Innate Immun 16, 265-272.

45. Buac K, Xu M, Cronin J, et al. (2009) NRG1/ERBB3 signaling in melanocyte development and melanoma: inhibition of differentiation and promotion of proliferation. Pigment Cell Melanoma Res 22, 773-784.

46. Zou XH, Jiang YZ, Zhang GR, et al. (2009) Specific interactions between human fibroblasts and particular chondroitin sulfate molecules for wound healing. Acta Biomater 5, $1588-1595$.

47. Offenhäuser N, Borgonovo A, Disanza A, et al. (2004) The eps8 family of proteins links growth factor stimulation to actin reorganization generating functional redundancy in the Ras/Rac pathway. Mol Biol Cell 15, 91-98.

48. Chapkin RS, McMurray DN \& Lupton JR (2007) Colon cancer, fatty acids and anti-inflammatory compounds. Curr Opin Gastroenterol 23, 48-54

49. Hatcher H, Planalp R, Cho J, et al. (2008) Curcumin: from ancient medicine to current clinical trials. Cell Mol Life Sci 65, 1631-1652.
50. Weaver KL, Ivester P, Seeds M, et al. (2009) Effect of dietary fatty acids on inflammatory gene expression in healthy humans. J Biol Chem 284, 15400-15407.

51. Gurtner GC, Werner S, Barrandon Y, et al. (2008) Wound repair and regeneration. Nature $\mathbf{4 5 3}, 314-321$.

52. Blikslager AT, Moeser AJ, Gookin JL, et al. (2007) Restoration of barrier function in injured intestinal mucosa. Physiol Rev 87, 545-564.

53. Barrientos S, Stojadinovic O, Golinko MS, et al. (2008) Growth factors and cytokines in wound healing. Wound Repair Regen 16, 585-601.

54. Rakoff-Nahoum S \& Medzhitov R (2007) Prostaglandinsecreting cells: a portable first aid kit for tissue repair. $J$ Clin Invest 117, 83-86.

55. Chapkin RS, Kim W, Lupton JR, et al. (2009) Dietary docosahexaenoic and eicosapentaenoic acid: emerging mediators of inflammation. Prostaglandins Leukot Essent Fatty Acids 81, 187-191.

56. Koeberle A, Northoff H \& Werz O (2009) Curcumin blocks prostaglandin $\mathrm{E}_{2}$ biosynthesis through direct inhibition of the microsomal prostaglandin $\mathrm{E}_{2}$ synthase-1. Mol Cancer Ther $\mathbf{8}, 2348-2355$.

57. Chakravarti N, Myers JN \& Aggarwal BB (2006) Targeting constitutive and interleukin-6-inducible signal transducers and activators of transcription 3 pathway in head and neck squamous cell carcinoma cells by curcumin (diferuloylmethane). Int J Cancer 119, 1268-1275.

58. Courtney ED, Matthews S, Finlayson C, et al. (2007) Eicosapentaenoic acid (EPA) reduces crypt cell proliferation and increases apoptosis in normal colonic mucosa in subjects with a history of colorectal adenomas. Int J Colorectal Dis 22, 765-776.

59. Fukata M, Michelsen KS, Eri R, et al. (2005) Toll-like receptor-4 is required for intestinal response to epithelial injury and limiting bacterial translocation in a murine model of acute colitis. Am J Physiol Gastrointest Liver Physiol 288, G1055-G1065.

60. Fukata M, Chen A, Klepper A, et al. (2006) Cox-2 is regulated by Toll-like receptor-4 (TLR4) signaling: role in proliferation and apoptosis in the intestine. Gastroenterology 131, 862-877.

61. Rakoff-Nahoum S \& Medzhitov R (2009) Toll-like receptors and cancer. Nat Rev Cancer 9, 57-63.

62. Wong SW, Kwon MJ, Choi AM, et al. (2009) Fatty acids modulate toll-like receptor 4 activation through regulation of receptor dimerization and recruitment into lipid rafts in a ros-dependent manner. J Biol Chem 284, 27384-27392.

63. Huang S, Zhao L, Kim K, et al. (2008) Inhibition of Nod2 signaling and target gene expression by curcumin. Mol Pharmacol 74, 274-281.

64. Lee DY, Lupton JR, Aukema HM, et al. (1993) Dietary fat and fiber alter rat colonic mucosal lipid mediators and cell proliferation. J Nutr 123, 1808-1817.

65. Buchanan FG \& DuBois RN (2006) Connecting COX-2 and Wnt in cancer. Cancer Cell 9, 6-8.

66. Hatazawa R, Tanaka A, Tanigami M, et al. (2007) Cyclooxygenase-2/prostaglandin $\mathrm{E}_{2}$ accelerates the healing of gastric ulcers via $\mathrm{EP}_{4}$ receptors. Am J Physiol Gastrointest Liver Physiol 293, G788-G797.

67. Ungaro R, Fukata M, Hsu D, et al. (2009) A novel Toll-like receptor 4 antagonist antibody ameliorates inflammation but impairs mucosal healing in murine colitis. Am J Physiol Gastrointest Liver Physiol 296, G1167-G1179.

68. Fan YY, Ly LH, Barhoumi R, et al. (2004) Dietary docosahexaenoic acid suppresses $\mathrm{T}$ cell protein kinase $\mathrm{C}$ theta lipid raft 
recruitment and IL-2 production. $J$ Immunol 173, 6151-6160.

69. Aggarwal S, Ichikawa H, Takada Y, et al. (2006) Curcumin (diferuloylmethane) down-regulates expression of cell proliferation and antiapoptotic and metastatic gene products through suppression of IkappaBalpha kinase and Akt activation. Mol Pharmacol 69, 195-206.

70. Nishihara M, Ogura H, Ueda N, et al. (2007) IL-6-gp130STAT3 in $\mathrm{T}$ cells directs the development of $\mathrm{IL}-17+\mathrm{Th}$ with a minimum effect on that of Treg in the steady state. Int Immunol 19, 695-702.

71. Wu S, Rhee KJ, Albesiano E, et al. (2009) A human colonic commensal promotes colon tumorigenesis via activation of $\mathrm{T}$ helper type $17 \mathrm{~T}$ cell responses. Nat Med 15, 1016-1022.

72. Kortylewski M, Xin H, Kujawski M, et al. (2009) Regulation of the IL-23 and IL-12 balance by Stat3 signaling in the tumor microenvironment. Cancer Cell 15, 114-123.

73. Bouwens M, van de Rest O, Dellschaft N, et al. (2009) Fishoil supplementation induces antiinflammatory gene expression profiles in human blood mononuclear cells. Am J Clin Nutr 90, 415-424.
74. Farzaneh-Far R, Harris WS, Garg S, et al. (2009) Inverse association of erythrocyte $n-3$ fatty acid levels with inflammatory biomarkers in patients with stable coronary artery disease: The Heart and Soul Study. Atherosclerosis 205, 538-543.

75. Mähler M, Bristol IJ, Leiter EH, et al. (1998) Differential susceptibility of inbred mouse strains to dextran sulfate sodiuminduced colitis. Am J Physiol 274, 3 Pt 1, G544-G551.

76. Suzuki R, Kohno H, Sugie S, et al. (2006) Strain differences in the susceptibility to azoxymethane and dextran sodium sulfate-induced colon carcinogenesis in mice. Carcinogenesis 27, 162-169.

77. Melgar S, Karlsson A \& Michaëlsson E (2005) Acute colitis induced by dextran sulfate sodium progresses to chronicity in $\mathrm{C} 57 \mathrm{BL} / 6$ but not in BALB/C mice: correlation between symptoms and inflammation. Am J Physiol Gastrointest Liver Physiol 288, G1328-G1338.

78. Uchiyama K, Nakamura M, Odahara S, et al. (2010) n-3 Polyunsaturated fatty acid diet therapy for patients with inflammatory bowel disease. Inflamm Bowel Dis 16, $1696-1701$. 